\title{
High-precision comparison of the antiproton-to-proton charge-to-mass ratio
}

\author{
S. Ulmer ${ }^{1}$, C. Smorra ${ }^{1,2}$, A. Mooser ${ }^{1}$, K. Franke ${ }^{1,3}$, H. Nagahama ${ }^{1,4}$, G. Schneider ${ }^{1,5}$, T. Higuchi ${ }^{1,4}$, S. Van Gorp ${ }^{6}$, K. Blaum ${ }^{3}$, \\ Y. Matsuda ${ }^{4}$, W. Quint ${ }^{7}$, J. Walz ${ }^{5,8}$ \& Y. Yamazaki ${ }^{6}$
}

Invariance under the charge, parity, time-reversal (CPT) transformation $^{1}$ is one of the fundamental symmetries of the standard model of particle physics. This CPT invariance implies that the fundamental properties of antiparticles and their matter-conjugates are identical, apart from signs. There is a deep link between CPT invariance and Lorentz symmetry - that is, the laws of nature seem to be invariant under the symmetry transformation of spacetimealthough it is model dependent ${ }^{2}$. A number of high-precision CPT and Lorentz invariance tests-using a co-magnetometer, a torsion pendulum and a maser, among others-have been performed ${ }^{3}$, but only a few direct high-precision CPT tests that compare the fundamental properties of matter and antimatter are available $e^{4-8}$. Here we report high-precision cyclotron frequency comparisons of a single antiproton and a negatively charged hydrogen ion $\left(\mathrm{H}^{-}\right)$carried out in a Penning trap system. From 13,000 frequency measurements we compare the charge-to-mass ratio for the antiproton $(q / m)_{\bar{p}}$ to that for the proton $(q / m)_{p}$ and obtain $(q / m)_{\bar{p}} /(q / m)_{p}-1=$ $1(69) \times 10^{-12}$. The measurements were performed at cyclotron frequencies of 29.6 megahertz, so our result shows that the CPT theorem holds at the atto-electronvolt scale. Our precision of 69 parts per trillion exceeds the energy resolution of previous antiproton-toproton mass comparisons ${ }^{7,9}$ as well as the respective figure of merit of the standard model extension ${ }^{10}$ by a factor of four. In addition, we give a limit on sidereal variations in the measured ratio of $<720$ parts per trillion. By following the arguments of ref. 11, our result can be interpreted as a stringent test of the weak equivalence principle of general relativity using baryonic antimatter, and it sets a new limit on the gravitational anomaly parameter of $\left|\alpha_{\mathrm{g}}-\mathbf{1}\right|<8.7 \times 10^{-7}$.

The standard model is the theory that describes particles and their fundamental interactions, although without taking into account gravitation. However, this model is known to be incomplete, which has inspired searches for physics beyond the standard model, such as tests of CPT invariance that compare the fundamental properties of matterto-antimatter equivalents at the lowest energies and with the greatest precision $^{12-15}$. For leptons, for example, the magnetic anomalies of electron and positron were compared with a fractional uncertainty of about 2 parts per billion ${ }^{4}$, and by applying similar techniques to protons and antiprotons, the resulting $g$-factor (a proportionality constant which links the spin of a particle to its magnetic moment) comparison reached a precision of 4.4 parts per million ${ }^{8}$. We are planning to improve this measurement by at least a factor of a thousand ${ }^{16,17}$. In this context, we recently reported the most precise and first direct high-precision measurement of the proton magnetic moment, with a fractional precision of 3.3 parts per billion ${ }^{18}$. Complementary to these efforts, spectroscopic comparisons of hydrogen and antihydrogen are underway; recent progress has been made at $\mathrm{CERN}^{13,19}$ The most precise test of CPT invariance with baryons is the comparison of the proton and antiproton charge-to-mass ratios. By measuring the cyclotron frequencies $v_{\mathrm{c}}=\left(q B_{0}\right) /(2 \pi m)$ of single trapped antiprotons and $\mathrm{H}^{-}$ions in a Penning trap with magnetic field $B_{0}$, the TRAP collaboration ${ }^{7}$ achieved a fractional precision of 90 parts per trillion.

In our measurements we also compare the cyclotron frequencies of a single antiproton and an $\mathrm{H}^{-}$ion. $\mathrm{H}^{-}$is used as a proxy for the proton; the negative charge facilitates the experiment by eliminating the need to invert trap voltages. Our advanced Penning trap system enables adiabatic particle exchange within $15 \mathrm{~s}$, which is much faster than in previous $(q / m)_{\mathrm{H}^{-} \bar{p}}$ comparisons. Our fast exchange rate allows for individual particle-to-antiparticle comparison cycles of only four minutes. This high-precision mass spectrometry method enabled us to perform about 6,500 direct frequency ratio comparisons within a total measuring time of 35 days. Moreover, our measurements have been carried out in thermal equilibrium with the detection system at 5.2(1.1) K, where systematic frequency shifts are small ${ }^{20}$.

Our cryogenic Penning-trap system, which consists of a measurement trap and a reservoir trap, is shown in Fig. 1. It is mounted in the horizontal bore of a superconducting magnet at $B_{0}=1.946 \mathrm{~T}$, the axis of the magnet being oriented at $60^{\circ}$ with respect to the Earth's rotation axis. Both traps have an inner diameter of $9 \mathrm{~mm}$ and are arranged in the five-electrode orthogonal and compensated design discussed in ref. 21. Transport electrodes connect the individual traps; they allow for fast adiabatic particle shuttling along the trap axis. The entire assembly is mounted in an indium-sealed cylindrical vacuum chamber with a volume of 1.2 litres. Once cooled to $4 \mathrm{~K}$, ultralow pressures are reached, and thus antiproton storage lifetimes of more than a year are achieved. To measure the particle's oscillation frequencies by image current detection ${ }^{22}$ we used highly sensitive superconducting resonant detection coils $\mathrm{s}^{23}$. The measurement trap detector is operated at a resonance frequency of $v_{\text {res }}=645,262 \mathrm{~Hz}$, has an inductance of $1.72 \mathrm{mH}$, and a quality factor of 11,300 .

Antiprotons are delivered in bunches by the Antiproton Decelerator of CERN; the antiproton bunches also release hydrogen from our degrader structure. $\mathrm{H}^{-}$ions are produced either by asymmetric dissociation of $\mathrm{H}_{2}$ or by electron capture (a detailed study of the production mechanism has yet to be performed). Typically 100 to 350 cold antiprotons and about a third that number of $\mathrm{H}^{-}$ions are prepared per ejection from the Antiproton Decelerator. From this particle cloud we extract a single antiproton and keep it in the centre of the trap, as well as an $\mathrm{H}^{-}$ion, which is parked in the downstream park electrode. The other particles are shuttled to the reservoir trap. In case particles are lost during experiments, the measurement trap is reloaded from this reservoir.

In charge-to-mass ratio comparisons cyclotron frequencies $v_{\mathrm{c}}$ are measured. We use the invariance theorem ${ }^{24}$

$$
v_{\mathrm{c}}^{2}=v_{+}^{2}+v_{z}^{2}+v_{-}^{2}
$$

which relates the characteristic trap frequencies $v_{+}, v_{-}$and $v_{\mathrm{z}}$ to $v_{\mathrm{c}}$. Comparisons of the antiproton-to- $\mathrm{H}^{-}$charge-to-mass ratio are 


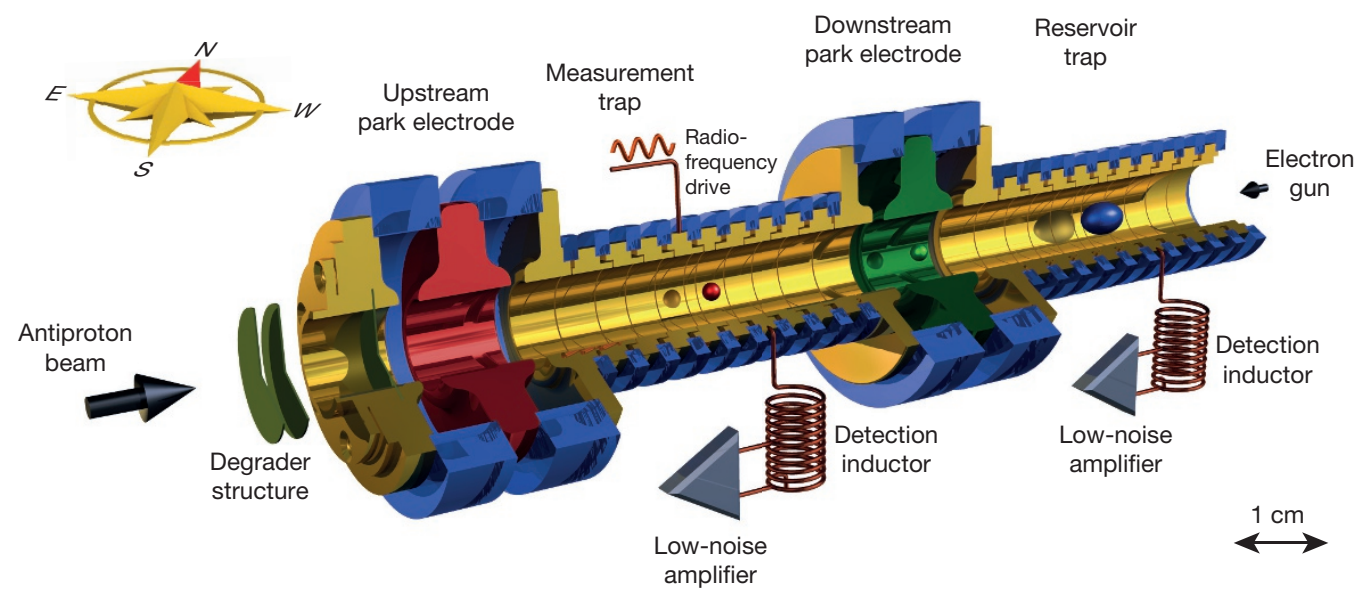

Figure $1 \mid$ Schematic of the measurement and the reservoir Penning traps. A resonant superconducting detection inductor is connected to each trap. Radio-frequency drives for particle manipulation are applied to the upstream correction electrode of the measurement trap. The upstream and downstream park electrodes are used for the particle shuttling scheme applied in the $\mathrm{q} / \mathrm{m}$

equivalent to a direct antiproton-to-proton comparison, but systematic shifts caused by polarity switching of the trapping voltages are avoided. The mass of the negative hydrogen ion is

$$
m_{\mathrm{H}^{-}}=m_{p}\left(1+2 \frac{m_{e}}{m_{p}}+\frac{\alpha_{\mathrm{pol}, \mathrm{H}^{-}} B_{0}^{2}}{m_{p}}-\frac{E_{\mathrm{b}}}{m_{p}}-\frac{E_{\mathrm{a}}}{m_{p}}\right)
$$

where $m_{e} / m_{p}$ is the electron-to-proton mass ratio ${ }^{25},\left(\alpha_{\text {pol, } \mathrm{H}^{-}} B_{0}^{2}\right) / m_{p}$ is the polarizability shift ${ }^{9}, E_{\mathrm{b}} / m_{\mathrm{p}}$ is the electron binding energy ${ }^{26}$ and $E_{\mathrm{a}} / m_{p}$ is the electron affinity of hydrogen ${ }^{27}$. If CPT invariance holds, the expected cyclotron frequency ratio is $R=\left(v_{\mathrm{c}}\right)_{\bar{p}} /\left(v_{\mathrm{c}}\right)_{\mathrm{H}^{-}}=$ $(q / m)_{\bar{p}} /(q / m)_{\mathrm{H}^{-}}=1.001089218754(2)$, the precision being limited by the accuracy of our knowledge of the proton mass ${ }^{28}$.

A detailed measurement cycle is shown in Fig. 2a. Our measurements are triggered by the antiproton injection into the Antiproton Decelerator; this avoids systematic ratio shifts induced by beats between the measurements and ambient field fluctuations caused by the Antiproton Decelerator cycle. Immediately after the injection trig-
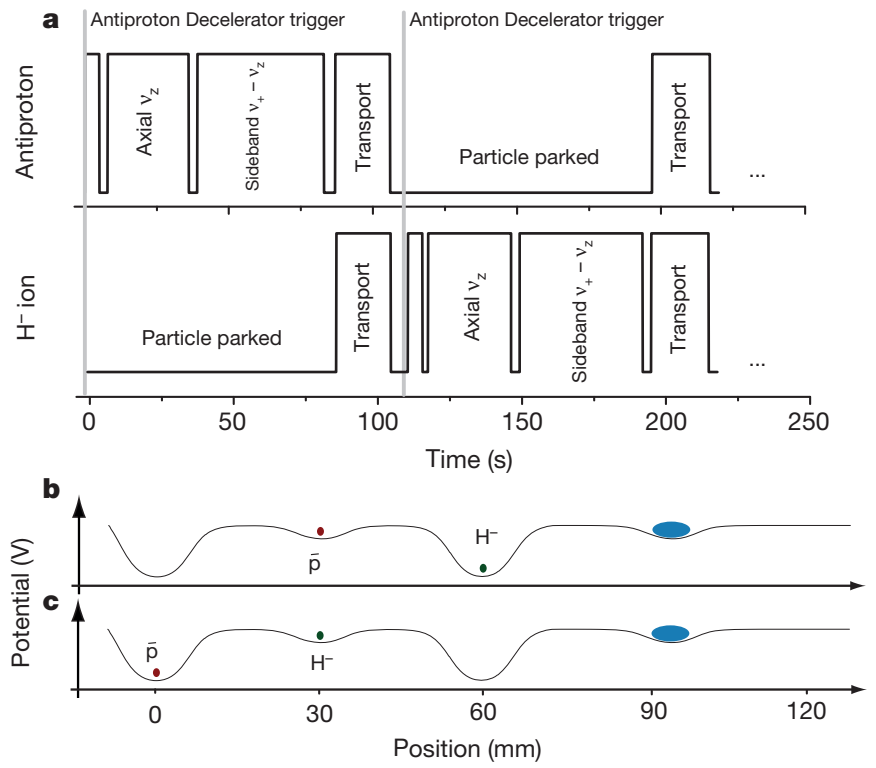

Figure $2 \mid$ Illustration of the measurement procedure. a, Detailed illustration of the measurement cycle. $\mathbf{b}$, Potential configuration for antiproton cyclotron frequency measurement. The blue ellipse represents the particle reservoir. c, Potential configuration for $\mathrm{H}^{-}$cyclotron frequency measurement. ratio comparisons. In addition, the degrader structure that slows down the 5.3$\mathrm{MeV}$ antiprotons from CERN's Antiproton Decelerator is shown on the left. The electron gun shown on the right provides particles for the electron cooling of antiprotons. The entire assembly is mounted in a cryogenic vacuum chamber (see text).

ger, the magnetron motion of the antiproton is cooled for $10 \mathrm{~s}$ (ref. 18). Then the antiproton's axial frequency $v_{z, \bar{p}}$ is measured, followed by a determination of the modified cyclotron frequency at $v_{+, \bar{p}}=29.656$ $\mathrm{MHz}$, which is extracted from a sideband measurement as described in ref. 18. These two measurements take $30 \mathrm{~s}$ and $48 \mathrm{~s}$, respectively. The particle's magnetron frequency is determined by evaluating $v_{-, \bar{p}} \approx v_{\mathrm{z}, \bar{p}}^{2} / 2 v_{+, \bar{p}}=7.02 \mathrm{kHz}$, and thus $v_{\mathrm{c}, \bar{p}} \approx 29.663 \mathrm{MHz}$ is obtained. Next, we ramp the trapping potential from the configuration shown in Fig. $2 \mathrm{~b}$ to that shown in Fig. $2 \mathrm{c}$, and adjust the trapping voltage by $5 \mathrm{mV}$ to tune the axial oscillation frequency of the $\mathrm{H}^{-}$ion into resonance with the superconducting detector. Afterwards, we perform similar measurements with the hydrogen ion to determine $v_{\mathrm{c}, \mathrm{H}^{-}} \approx 29.635 \mathrm{MHz}$. Thus, a single ratio comparison takes exactly two Antiproton Decelerator cycles, corresponding to a typical measuring time of 220-240 s. To obtain from all performed measurements the final experimentally determined frequency ratio $R_{\exp }=v_{\mathrm{c}, \bar{p}} / v_{\mathrm{c}, \mathrm{H}^{-}}=$ $(q / m)_{\bar{p}} /(q / m)_{\mathrm{H}^{-}}$, we reject those data points measured when magnetic field changes caused by activities in the Antiproton Decelerator accelerator hall were observed. These changes are identified by an array of giant magneto resistance, Hall and flux-gate magnetic field sensors.

Subsequently the ratios are processed as follows. To remove systematic ratio shifts caused by the intrinsic magnetic-field drift $1 / B_{0} \times(\Delta B / \Delta t)=-5(1) \times 10^{-9}$ per hour of our superconducting magnet, we compute the antiproton cyclotron frequencies $\left(v_{c, \bar{p}, k}\right)(t)=$ $v_{\mathrm{c}, \bar{p}, k}+\left(v_{\mathrm{c}, \bar{p}, k+1}-v_{\mathrm{c}, \bar{p}, k}\right) /\left(t_{k+1}-t_{k}\right) t$, where $k$ is the index of individual measurements. Subsequently we evaluate the upper ratio $\left(v_{\mathrm{c}, \bar{p}, k}\right)\left(t_{\mathrm{H}^{-}, k}\right) / v_{\mathrm{c}, \mathrm{H}^{-}, k}$, where $t_{\mathrm{H}^{-}, k}$ is the centre time of the $v_{\mathrm{c}, \mathrm{H}^{-}, k}$ determination. In addition, the reciprocal ratios $v_{\mathrm{c}, \bar{p}, k} /\left(v_{\mathrm{c}, \mathrm{H}^{-}, k}\right)\left(t_{\bar{p}, k}\right)$ are evaluated in a similar way. From the processed data we extract the mean of the frequency ratio by performing a maximum-likelihood fit of a Gaussian distribution to the upper ratio as well as to the reciprocal results and calculating the average of both.

To estimate the uncertainty of the mean we evaluate the correlation matrix of the extracted ratios and calculate the standard error of the cross-correlated data. This avoids underestimation of the error caused by frequencies which are, owing to the linear averaging approach, used in multiple ratios. All measured upper ratios are shown in Fig. 3a and b. The full time sequence is shown in Fig. $3 \mathrm{a}$, and the results projected to a histogram are shown in Fig. 3b. Breaks in the time sequence are due to maintenance of the apparatus or systematic measurements. In Fig. $3 \mathrm{c}$ and $\mathrm{d}$, the power spectrum density and the Allan deviation of all measured data points are shown. The mean of the power spectrum density is constant, while a linear fit to the double-log plot of the Allan 

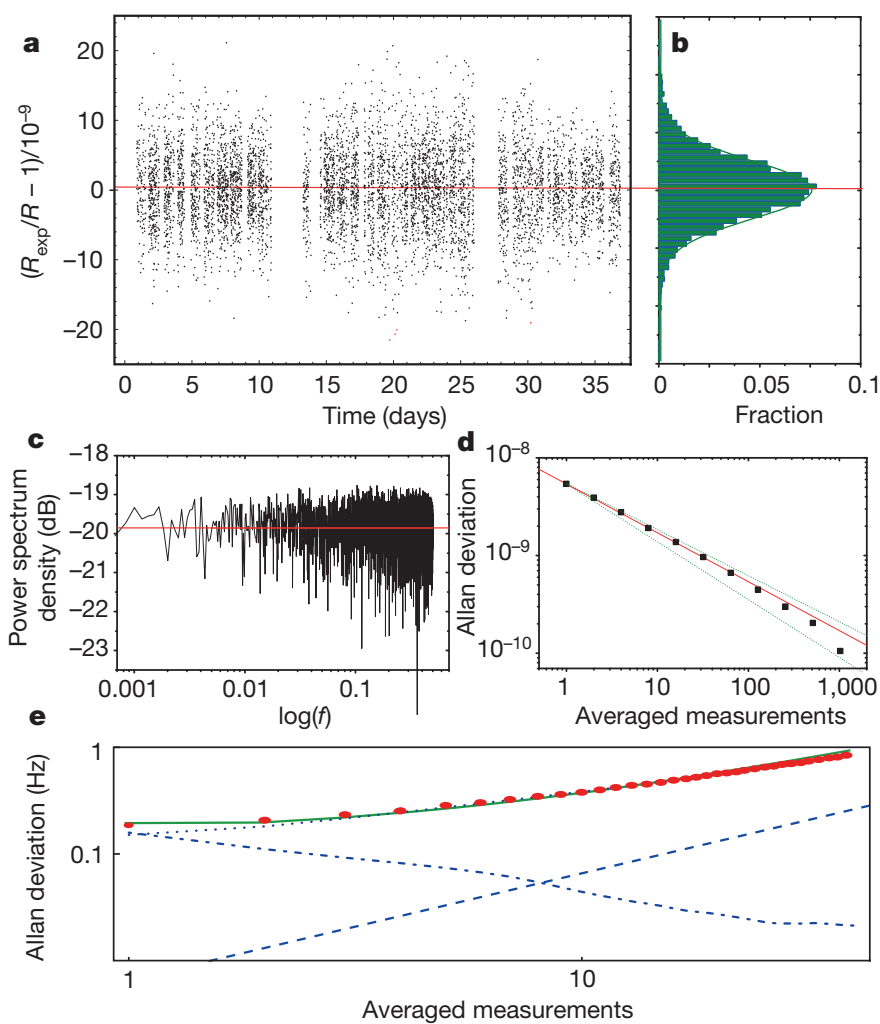

Figure $3 \mid$ Results and data analysis. a, All measured antiproton-to- $\mathrm{H}^{-}$ cyclotron frequency ratios as a function of time. Within 35 days, 6,521 frequency ratios were measured. Fraction, fraction of total counts (number measured in one bin normalized to the number of all measurements). $\mathbf{b}$, Measured ratios projected to a histogram. c, Power spectrum density of the ratios as a function of reciprocal measurements. $\mathbf{d}$, Allan deviation of the measured ratios including a fit to the data with slope $-0.501(2)$, which confirms that the ratio fluctuations follow a Gaussian white noise distribution. e, Allan deviation of cyclotron frequency measurements. Details are discussed in the text.

deviation gives a slope of $\alpha=-0.501(2)$. This confirms the Gaussian white-noise nature of the ratio fluctuations and justifies our data analysis.

For further verification we evaluate the Allan deviation of our cyclotron frequency measurements, which is shown in Fig. $3 e$ as the red data points. A fit of a linear drift of -5(1) parts per billion per hour (dashed line in Fig. 3e), a white-noise contribution with a root-mean-square width of $160(15) \mathrm{mHz}$ per cycle (dash-dotted line in Fig. 3e), and a random walk generating a Gaussian distribution of $220(20) \mathrm{mHz}$ per cycle (dotted line in Fig. $3 \mathrm{e}$ ) reproduce the experimental results. From this model we simulate data, add random offsets $\Delta_{r}$ and blind-analyse the simulation data. Within error bars the offsets $\Delta_{r}$ are reproduced, which independently justifies our data analysis. The antiproton-to- $\mathrm{H}^{-}$ mass ratio extracted from this data evaluation is

$$
R_{\exp }=1.001089218872(64)
$$

To further check both the data analysis and the experiment we also evaluate the cyclotron frequency ratios for $\bar{p}$-to- $\bar{p}$ and $\mathrm{H}^{-}$-to- $\mathrm{H}^{-}$ instead of $\bar{p}$-to- $\mathrm{H}^{-}$, but measured within subsequent cycles. For these direct comparisons of identical particles

$$
R_{\text {exp,id }}-1=-3(79) \times 10^{-12}
$$

is obtained, which is consistent with 1 . The increased statistical error in the second case is caused by the random walk in the magnetic field, which leads to slightly higher ratio fluctuations owing to the doubled time interval between subsequent measurements of identical particles.

Several systematic corrections enter into the measured antiprotonto- $\mathrm{H}^{-}$charge-to-mass ratio $R_{\exp }$. The dominant systematic shift is related to particle exchange and the $5-\mathrm{mV}$ detuning of the trapping voltage, which is required to tune the axial oscillation frequencies of both particles to the centre of the axial detector. Slightly different contact and offset potentials as well as machining imperfections are present at each individual trap electrode. Thus, the change of the ring voltage causes a relative shift of the antiproton-to- $\mathrm{H}^{-}$equilibrium position. In the presence of a magnetic gradient term of $B_{1}=7.58(42) \mathrm{mT} \mathrm{m}^{-1}$ (where $B_{1}$ is the strength of the magnetic gradient) the cyclotron frequencies $v_{\mathrm{c}, \bar{p}}$ and $v_{\mathrm{c}, \mathrm{H}^{-}}$are hence measured at slightly different magnetic fields, leading to a systematic ratio shift. In our measurement, the adjustment of the trapping voltage shifts the $\mathrm{H}^{-}$ion towards lower magnetic fields, and $R_{\exp }$ has to be corrected by $-114(26)$ parts per trillion. A detailed discussion of this dominant systematic shift is provided in the Supplementary Information.

The systematic uncertainty arises from the uncertainty in the determination of the offset voltages and the magnetic gradient $B_{1}$. In addition to the particle position, the magnitude of the octupolar correction $C_{4}$ of the trapping potential ${ }^{20}$ also changes when the trap voltage is adjusted. The resulting ratio correction is at $-3(1)$ parts per trillion, the error being due to uncertainties extracted from potential theory and the determination of the axial mode temperature $T_{\mathrm{z}}$ of the particle. Eventually, the stability of our rubidium frequency reference contributes a systematic scatter of 3 parts per trillion per ratio comparison. In summary, the ratio has to be corrected by $-117(26)$ parts per trillion, leading to our final result

$$
R_{\text {exp,c }}=1.001089218755(64)(26)
$$

which corresponds to an antiproton-to-proton mass ratio of

$$
\frac{(q / m)_{\bar{p}}}{(q / m)_{p}}-1=1(64)(26) \times 10^{-12}
$$

and is in agreement with CPT conservation.

In the framework of the standard model extension developed by ref. 29 a figure of merit $r^{\mathrm{H}^{-}}=\left(1-R_{\text {exp,c } \mathrm{c}}\right) h v_{\mathrm{c}, \mathrm{H}^{-}} / m_{\mathrm{H}^{-}} c^{2}$ is derived, which characterizes the sensitivity of our measurement with respect to the CPT-violating terms added to the standard-model Lagrange density. Our result sets a new limit of $r^{\mathrm{H}^{-}}<9 \times 10^{-27}$. This exceeds the previous limit by a factor of four, and probes the standard model at an energy scale of 8 atto-electronvolts. In terms of energy sensitivity our result is the most stringent test of CPT invariance with baryonic antimatter performed so far.

In addition to the above discussion, our high data accumulation rate enables the search for sidereal variations, which might be mediated by cosmological background fields. To this end, the data set is processed using lock-in filter and Allan-deviation-based data analysis. A diurnal variation of the results would appear as a peak in the lock-in spectrum at a period of the sidereal day, which is $86,164.1 \mathrm{~s}$. We do not observe such an indication and from our data analysis we conclude that the amplitude of any diurnal variation in $R_{\exp }$ is $<0.72$ parts per billion at the 0.95 confidence level.

By following the arguments of ref. 11 our measurements can be interpreted as a test of weak equivalence. If the proton-to-antiproton charge-to-mass ratios are identical in the absence of a gravitational field, then the particle and antiparticle cyclotron clocks have the same frequencies. However, if matter respects weak equivalence while antimatter experiences an anomalous coupling to the gravitational field, the cyclotron frequencies of the two particles will experience different gravitational redshifts when moved to the surface of the Earth. We follow ref. 11, in which a possible gravitational anomaly acting on antimatter is expressed by a parameter $\alpha_{\mathrm{g}}$, which modifies the effective newtonian gravitational potential $U$ to give $\alpha_{\mathrm{g}} U$. This contributes a possible difference in the measured cyclotron frequencies of $\left(v_{c, p}-v_{c, \bar{p}}\right) /\left(v_{c, p}\right)=-3\left(\alpha_{g}-1\right) U / c^{2}$ Thus, our measurement sets a new upper limit of $\left|\alpha_{\mathrm{g}}-1\right|<8.7 \times 10^{-7}$. 


\section{Received 26 May; accepted 17 June 2015.}

1. Lüders, G. Proof of the TCP theorem. Ann. Phys. 2, 1-15 (1957).

2. Greenberg, O. W. CPT violation implies Lorentz violation. Phys. Rev. Lett. 89, 231602 (2002)

3. Kostelecký, V. A. \& Russell, N. Data-tables on Lorentz and CPT Violation. Rev. Mod. Phys. 83, 11-31 (2011).

4. Van Dyck, R., Jr, Schwinberg, P. \& Dehmelt, H. New high-precision comparison of electron and positron g factors. Phys. Rev. Lett. 59, $26-29$ (1987).

5. Schwingenheuer, B. etal. CPT tests in the neutral Kaon system. Phys. Rev. Lett. 74, 4376-4379 (1995)

6. Miller, J. P., De Rafael, E. \& Roberts, B. L. Muon (g-2): experiment and theory. Rep. Prog. Phys. 70, 795 (2007)

7. Gabrielse, G. etal. Precision mass spectroscopy of the antiproton and proton using simultaneously trapped particles. Phys. Rev. Lett. 82, 3198-3201 (1999).

8. DiSciacca, J. et al. One-particle measurement of the antiproton magnetic moment. Phys. Rev. Lett. 110, 130801 (2013).

9. Thompson, J. K., Rainville, S. \& Pritchard, D. E. Cyclotron frequency shifts arising from polarization forces. Nature 430, 58-61 (2004).

10. Colladay, D. \& Kostelecký, V. A. CPT violation and the standard model. Phys. Rev. D 55, 6760-6774 (1997).

11. Hughes, R. J. \& Holzscheiter, M. H. Constraints on the gravitational properties of antiprotons and positrons from cyclotron-frequency measurements. Phys. Rev. Lett. 66, 854-857 (1991).

12. Hori, M. etal. Two-photon spectroscopy of antiprotonic helium and the electron-toantiproton mass ratio. Nature 475, 484-488 (2011).

13. Kuroda, N. et al. A source of antihydrogen atoms for in flight hyperfine spectroscopy. Nature Commun. 5, 3089-3092 (2014).

14. Andresen, G. B. et al. Confinement of antihydrogen for 1,000 seconds. Nature Phys. 7, 558-564 (2011)

15. Gabrielse, G. et al. Background-free observation of cold antihydrogen and a fieldionization analysis of its states. Phys. Rev. Lett. 89, 213401 (2002).

16. Ulmer, S. et al. CERN Document Server Report SPSC-TDR-002, http://cds.cern.ch/ record/1503514? In5de (CERN, 2013).

17. Smorra, C. et al. Towards a high-precision measurement of the antiproton magnetic moment. Hyperfine Interact. 228, 31-36 (2014)

18. Mooser, A. et al. High-precision measurement of the magnetic moment of the proton. Nature 509, 596-599 (2014).

19. Amole, C. et al. Resonant quantum transitions in trapped antihydrogen atoms. Nature 483, 439-443 (2012).

20. Brown, L. S. \& Gabrielse, G. Geonium theory: physics of a single electron or ion in a Penning trap. Rev. Mod. Phys. 58, 233-311 (1986).

21. Gabrielse, G., Haarsma, L. \& Rolston, L. S. Open-endcap Penning traps for high precision experiments. Int. J. Mass Spectrom. 88, 319-332 (1989).

22. Wineland, D. J. \& Dehmelt, H. G. The stored ion calorimeter. J. Appl. Phys. $\mathbf{4 6}$, 919-922 (1975).
23. Ulmer, $S$. et al. The quality factor of a superconducting resonator in a magnetic field. Rev. Sci. Instrum. 80, 123302 (2009).

24. Brown, L. S. \& Gabrielse, G. Precision spectroscopy of a charged particle in an imperfect Penning trap. Phys. Rev. A 25, 2423-2425 (1982)

25. Sturm, S. et al. High-precision measurement of the atomic mass of the electron. Nature 506, 467-470 (2014).

26. Parthey, C. G. et al. Improved measurement of the hydrogen 1S-2S transition frequency. Phys. Rev. Lett. 107, 203001 (2011).

27. Bhatia, A. K. \& Drachmann, R. J. Polarizability of helium and the negative hydrogen ion. J. Phys. At. Mol. Opt. Phys. 27, 1299-1305 (1994).

28. Van Dyck, R. S. Jr, Farnham, D. L., Zafonte, S. L. \& Schwinberg, P. B. High precision Penning trap mass spectroscopy and a new measurement of the proton's atomic mass. AlP Conf. Proc. 457, 101-110 (1999).

29. Bluhm, R., Kostelecký, V. A. \& Russell, N. CPT and Lorentz tests in Penning traps. Phys. Rev. D 57, 3932-3943 (1998)

Supplementary Information is available in the online version of the paper.

Acknowledgements We acknowledge support by the Antiproton Decelerator group and all other CERN groups which provide support to Antiproton Decelerator experiments. We thank L. Bojtar and F. Butin for their invaluable support. We acknowledge financial support by the RIKEN Initiative Research Unit Program, RIKEN President Funding, RIKEN Pioneering Project Funding, RIKEN FPR Funding, the RIKEN JRA Program, the Grant-in-Aid for Specially Promoted Research (grant number 24000008) of MEXT, the Max-Planck Society, the IMPRS-PTFS, the EU (ERC advanced grant number 290870-MEFUCO), the BMBF, the Helmholtz-Gemeinschaft, and the CERN Fellowship programme.

Author Contributions S.U., C.S. and G.S. designed the apparatus which was then developed and commissioned by S.U., C.S., G.S., K.F., H.N., T.H. and A.M.; S.U., A.M. and C.S. developed the software system and experiment control. S.U., A.M., C.S., H.N., T.H., K.F. and G.S. participated in the 2014 antiproton run and contributed to the data collection. C.S., A.M., and S.U. discussed and analysed the data. S.U. and A.M. performed the systematic studies. The manuscript was written by S.U., A.M., K.B. and J.W. and then discussed and approved by all authors.

Author Information Reprints and permissions information is available at www.nature.com/reprints. The authors declare no competing financial interests. Readers are welcome to comment on the online version of the paper. Correspondence and requests for materials should be addressed to S.U. (stefan.ulmer@cern.ch).

(c) (i) (2) This work is licensed under a Creative Commons Attribution-

cc) $19 \mathrm{sy}$ NonCommercial-ShareAlike 3.0 Unported licence. The images or other third party material in this article are included in the article's Creative Commons licence, unless indicated otherwise in the credit line; if the material is not included under the Creative Commons licence, users will need to obtain permission from the licence holder to reproduce the material. To view a copy of this licence, visit http://creativecommons. org/licenses/by-nc-sa/3.0 